\title{
O renascimento da terapia psicodélica: Uma revisão integrativa da literatura
}

\author{
The rebirth of psychedelic therapy: An integrative literature review \\ El renacimiento de la terapia psicodélica: Una revisión integradora de la literatura
}

Recebido: 11/07/2021 | Revisado: 17/07/2021 | Aceito: 24/07/2021 | Publicado: 31/07/2021

\author{
Henrique da Cunha Santos \\ ORCID: https://orcid.org/0000-0002-5665-990X \\ Centro Universitário de Patos, Brasil \\ E-mail: henriquesantos@med.fiponline.edu.br \\ Cássio Ilan Soares Medeiros \\ ORCID: https://orcid.org/0000-0002-6413-8303 \\ Centro Universitário de Patos, Brasil \\ E-mail: medeiroscassio4@gmail.com
}

\begin{abstract}
Resumo
Objetivo: O presente estudo é uma abordagem geral sobre a ciência psicodélica e tem por objetivo, a partir de uma revisão integrativa da literatura, contextualizar o renascimento da terapia psicodélica. Metodologia: Por meio da pesquisa na base de dados eletrônica Medical Publications (PubMed) e na Scientific Eletronic Library (Scielo) com o descritor em inglês: "psychedelic therapy" foram selecionados os artigos que compõem esse trabalho. A pesquisa foi realizada entre janeiro e maio de 2021. Os critérios de inclusão utilizados foram artigos em inglês e em português, publicados nos últimos 20 anos e relacionados com a questão de pesquisa. Resultados: Foram encontrados 43 artigos na pesquisa nas bases de dados. Entretanto apenas 7 artigos foram selecionados para análise de acordo com os critérios de inclusão. Conclusão: Após análise dos textos foi possível correlacionar vários aspectos e concluir que há grandes perspectivas e potencialidades na terapia psicodélica e que o seu renascimento se dá num contexto de transformações sociais e de demandas na psicoterapia não atendidas.
\end{abstract}

Palavras-chave: Terapia; Renascimento; Psicodélico; Alucinógenos.

\begin{abstract}
Objective: This study is a general approach to psychedelic science and aims, from an integrative literature review, to contextualize the rebirth of psychedelic therapy. Methodology: By searching the electronic database Medical Publications (PubMed) and the Scientific Electronic Library (Scielo) with the descriptor in English: "psychedelic therapy", the articles that make up this work were selected. The research was conducted between January and May 2021. The inclusion criteria used were articles in English and Portuguese, published in the last 20 years and related to the research question. Results: 43 articles were found in the search in the databases. However, only 7 articles were selected for analysis according to the inclusion criteria. Conclusion: After analyzing the texts, it was possible to correlate several aspects and conclude that there are great perspectives and potentials in psychedelic therapy and that its rebirth takes place in a context of social transformations and unmet demands in psychotherapy.
\end{abstract}

Keywords: Therapy; Rebirth; Psychedelic; Hallucinogens.

\section{Resumen}

Objetivo: Este estudio es un acercamiento general a la ciencia psicodélica y tiene como objetivo, a partir de una revisión integradora de la literatura, contextualizar el renacimiento de la terapia psicodélica. Metodología: Mediante la búsqueda en la base de datos electrónica Medical Publications (PubMed) y en la Scientific Electronic Library (Scielo) con el descriptor en inglés: "terapia psicodélica", se seleccionaron los artículos que componen este trabajo. La investigación se realizó entre enero y mayo de 2021. Los criterios de inclusión utilizados fueron artículos en inglés y portugués, publicados en los últimos 20 años y relacionados con la pregunta de investigación. Resultados: se encontraron 43 artículos en la búsqueda en las bases de datos. Sin embargo, solo se seleccionaron 7 artículos para el análisis según los criterios de inclusión. Conclusión: Luego de analizar los textos, fue posible correlacionar varios aspectos y concluir que existen grandes perspectivas y potencialidades en la terapia psicodélica y que su renacimiento se da en un contexto de transformaciones sociales y demandas insatisfechas en psicoterapia.

Palabras clave: Terapia; Renacimiento; Psicodélico; Alucinógenos. 


\section{Introdução}

Em 1938 na Suíça, em meio à Segunda guerra mundial, o químico Albert Hofmann, procurava por medicamentos para a circulação sanguínea e aparelho respiratório quando sintetizou pela primeira vez a Dietilamida do ácido lisérgico - o LSD. Apenas 5 anos depois viria à tona, em um auto experimento de Hofmann, as suas propriedades psíquicas (Hofemann, 2013).

Pouco depois dos experimentos em animais, foi feito, em 1947, o primeiro teste sistemático em humanos. Com a divulgação dos resultados, não demorou a surgir o interesse psiquiátrico e terapêutico na substância que era descrita como o psicoativo mais potente e interessante até então conhecido (Hofemann, 2013).

Seu efeito profundo e misterioso também chamou a atenção do meio artístico e intelectual, nos quais se deram os primeiros usos não medicinas do alucinógeno. Quase simultaneamente, por volta da metade dos anos 50, foram redescobertos os "cogumelos sagrados" e o seu princípio ativo, a psilocibina - uma molécula muita semelhante ao LSD, isolado. Eles eram utilizados há milênios em rituais religiosos e contextos sagrados na América central (Hofemann, 2013).

Ao final da década de 50, essas substâncias estavam estabilizadas no cenário psiquiátrico e psicoterapêutico dos Estados unidos e, em menor parte, da Europa (Doblim, 2000). Somente nos anos 60 o uso recreativo e não terapêutico dessas drogas se difundiu entre a população (Brasil, 2021). Elas eram usadas, na sua maioria, por Hippies e pacifistas que pediam o fim da Guerra do Vietnã (Leary, 1983). Não demorou muito para que a onda de protestos pacifistas e o uso indiscriminado dessas substâncias levasse, em 1970, a ilegalização do LSD nos Estados Unidos da América (EUA). Assim como esse fato, também ocorreu o mesmo com a pesquisa e a psicoterapia em alucinógenos (Daniel \& Haberman, 2017).

Por mais de 20 anos, a discriminação dessas substâncias impossibilitou estudos científicos com alucinógenos (Strassman et al., 1994, 1996; Strassman, 1996, 2001). A partir dos anos 1990, com algumas poucas autorizações especiais, voltaram à tona estudos neurológicos com essas drogas e, principalmente, o que agora tem sido chamado de terapias psicodélicas (James, 2017).

Após duas décadas de quiescência, a pesquisa psicodélica clínica foi reiniciada na década de 1990 e é rapidamente progressiva. As primeiras evidências de eficácia são promissoras, mas a compreensão dos processos psicológicos de mudanças subjacentes aos benefícios observados ainda é limitada (Luoma, Sabucedo, Eriksson, Gates \& Pilecki, 2019).

A terapia psicodélica assistida compreende três estágios: preparação, sessão psicodélica e integração. A preparação é a chave para maximizar o potencial do benefício de um psicodélico sendo a experiência e integração importantes para prolongar as melhorias. O modelo psicológico de flexibilidade (PFM) parece ser promissor para orientar a preparação psicodélica e sua integração. Tendo isso em mente, um novo modelo baseando-se na aceitação, conexão e incorporação, apresenta os seis processos psicológicos de flexibilidade, renomeados e reorganizados em uma tríade de aceitação (desfusão cognitiva, foco no momento presente e vontade) e uma tríade de conexão (o eu como contexto, valores comprometidos e ação) (Watts \& Luoma, 2020).

A confluência desses seis processos é a flexibilidade psicológica, ou a capacidade de entrar em contato com o momento presente mais plenamente como um ser humano consciente e, com base no que a situação permite, a capacidade para mudar ou persistir no comportamento a fim de servir a fins valiosos. Além disso, diversos estudos têm demonstrado que melhorias na flexibilidade psicológica está relacionada com melhores desfechos clínicos (Watts \& Luoma, 2020).

Esse método incorpora ainda suporte de psicoterapia que normalmente consiste em um período de preparação, seguido por uma dose moderada a alta de psicodélicos na presença de um ou mais terapeutas, seguido por uma ou mais sessões de integração (Luoma, Sabucedo, Eriksson, Gates \& Pilecki, 2019). 
A terapia psicodélica oferece um caminho para a consciência incorporada, possibilitando alívio e aprendizado aos pacientes. Os psicodélicos também oferecem a oportunidade para mais pacientes experimentarem profundas mudanças psicológicas do que apenas através da terapia da fala, sendo uma boa terapia adjuvante (Watts \& Luoma, 2020).

Transtornos do humor (por exemplo, depressão, ansiedade, transtorno do estresse pós-traumático - TEPT), álcool e outros transtornos por uso de drogas representam um grande fardo para o indivíduo, famílias e sistemas de saúde, com significantes gastos de saúde pública. Psicodélicos como o LSD e a psilocibina podem representar novas opções de tratamento para transtornos do humor e transtornos do uso de álcool e outras drogas (Gardner, Carter, O’brien \& Seear, 2019).

Os medicamentos psicodélicos incluem compostos como psilocibina, dietilamida de ácido lisérgico (LSD) e ayahuasca, que induzem estados alterados de consciência agindo nos receptores 5-HT2A do cérebro (ou seja, esses compostos convencionalmente definidos como "psicodélico"), e também 3,4-metilenodioximetanfetamina (MDMA) que tem efeitos de "alteração da mente" por meio de um caminho neuroquímico diferente (Gardner, Carter, O’brien \& Seear, 2019).

O atual ressurgimento da importância sobre a eficácia desses compostos está no seu início, mas as descobertas preliminares são promissoras: alguns benefícios foram demonstrados para indicações como transtorno de depressão maior, transtorno de estresse pós-traumático (TEPT), transtorno obsessivo-compulsivo (TOC), ansiedade associada a doenças com risco de vida, dependência ao álcool, tabagismo a longo prazo, sendo os riscos identificados de toxicidade e dependência considerados baixos (Gardner, Carter, O’brien \& Seear, 2019).

Nesse contexto, faz-se digna uma investigação das circunstâncias desse fenômeno, uma vez que este tem se mostrado promissor em uma área que carece de tratamentos definitivos. A fim nortear essa discussão, o objetivo do presente estudo é o de contextualizar o renascimento da terapia psicodélica no cenário da prática clínica atual, além de explicar como a terapia psicodélica ressurgiu no cenário clínico, psiquiátrico e psicoterapêutico, por meio da seguinte questão de pesquisa: "Qual a finalidade da terapia psicodélica no âmbito psiquiátrico e psicoterapêutico?". A justificativa para a escolha do tema faz-se pela relevância no cenário clínico desempenhada por essa terapia e seus benefícios no manejo dos pacientes.

\section{Metodologia}

Esse estudo se trata de uma revisão integrativa da literatura, tipo de estudo que permite um mapeamento do conteúdo produzido sobre uma temática específica, possibilita sintetizar os principais resultados dos estudos, auxiliando a tomada de decisão frente ao paciente e na identificação de lacunas para realização de pesquisas futuras (Souza, Silva \& Carvalho, 2010).

Para a elaboração desta revisão integrativa da literatura adotou-se as seguintes etapas: estabelecimento da pergunta norteadora; objetivos da revisão integrativa; estabelecimento de critérios de inclusão e exclusão de artigos (seleção da amostra); definição das informações a serem extraídas dos artigos selecionados; análise dos resultados; discussão e apresentação dos resultados (Souza, Silva \& Carvalho, 2010).

1 - Em um primeiro momento, definiu-se a questão de pesquisa que norteou a pesquisa nas bases de dados, sendo ela: ““'Qual a finalidade da terapia psicodélica no âmbito psiquiátrico e psicoterapêutico?”. A partir disso procedeu-se com a realização das demais etapas.

2 - Definiram-se os objetivos dessa revisão integrativa, que foram: mostrar de que forma se dá o renascimento da terapia psicodélica; investigar as causas e impulsionadores desse renascimento; abordar as finalidades das terapêuticas desenvolvidas e o objetivo dos envolvidos; explicar como a terapia psicodélica ressurgiu no cenário clínico psiquiátrico e psicoterapêutico atuais.

3 - Após isso, foram selecionados os critérios de inclusão (artigos em inglês ou português), artigos publicados nos últimos 20 anos e que estivessem relacionados diretamente com a questão de pesquisa, e os critérios de exclusão (artigos 
repetidos, não relacionados com a questão de pesquisa e por se encontrarem indisponíveis para leitura). Essa etapa foi fundamental para a pesquisa nas bases de dados Medical Publisher (PUBMED) e na Scientific Eletronic Library (Scielo). O período que compreendeu as buscas foi entre janeiro e maio de 2021.

4 - Definição das informações a serem extraídas dos artigos selecionados: aqui, realizou-se a leitura e interpretação das principais informações dos artigos escolhidos para compor a amostra final.

5 - Após isso, a próxima etapa foi a análise das informações encontradas, extraindo-se as informações diretamente relacionadas com a questão de pesquisa e sistematizando-as nos quadros 2,3 e 4 .

6 - Com isso procedeu-se com a construção da argumentação para defender a questão de pesquisa e os resultados foram apresentados e contextualizados.

Para a seleção dos artigos foram utilizadas as bases de dados PUBMED e Scientific Eletronic Library (Scielo). Entretanto, não foram identificados artigos relacionados com a questão de pesquisa na Scielo, sendo a PUBMED considerada suficientemente ampla para o propósito do estudo.

Para os critérios de inclusão, optou-se por artigos publicados nos últimos 20 anos, tendo em vista a baixa quantidade de estudos relacionados com a questão de pesquisa, utilizou-se como descritor: "psychedelic therapy", com o qual se obteve 43 artigos que, quando filtrados pelo período de 2000 a 2020, resultaram em um total de 40. Assim, com base no tema proposto, a partir dos critérios de exclusão e de artigos de pesquisa relacionados diretamente com a questão de pesquisa, foram selecionados 7 artigos a fim de abranger a pergunta norteadora.

Figura 1 - Fluxograma de seleção dos artigos.

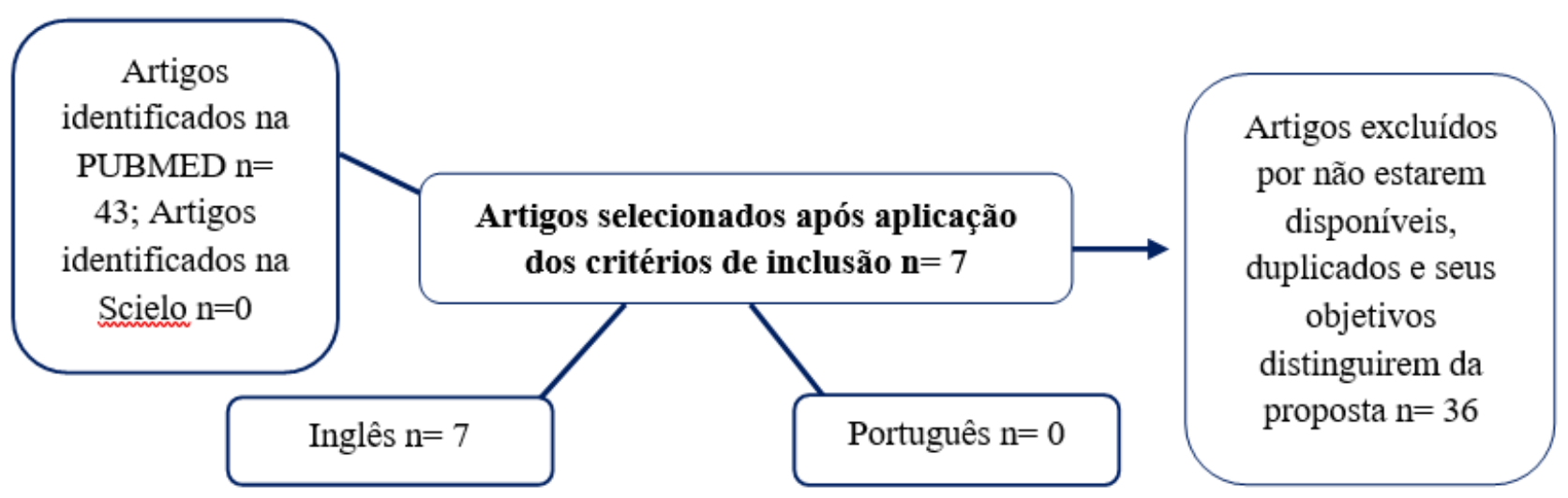

Fonte: Dados da pesquisa (2021).

Os estudos encontrados foram organizados em uma planilha no software Excel com a descrição do título dos estudos, essa ferramenta possibilitou a remoção de duplicadas. Em seguida, realizou-se uma leitura criteriosa dos títulos e resumos para verificar quais estudos estavam com a pergunta norteadora dessa pesquisa, a partir disso foi analisada sua adequação aos demais critérios de seleção desse estudo que foram descritos acima. Por fim, foi realizada a leitura na íntegra da amostra final e extraídos os dados desejados.

\section{Resultados}

Os estudos selecionados foram traduzidos para o português e subdivididos em dois quadros. O primeiro quadro classificou os artigos selecionados de acordo com as seguintes informações: autor/ano, título e objetivo geral do estudo. Após 
a análise dos dados, foi possível observar que os anos de 2017 e 2019 foram os que apresentaram 1 artigo em cada ano. Com maior número de publicações está o ano de 2018 com um total de 5 artigos.

Quadro 1: Distribuição dos estudos analisados quanto aos autores/ano, título e objetivo geral.

\begin{tabular}{|c|c|c|c|}
\hline $\mathbf{N}^{\circ}$ & $\begin{array}{c}\text { AUTOR/ } \\
\text { ANO }\end{array}$ & TÍTULO & OBJETIVO GERAL \\
\hline 1 & $\begin{array}{l}\text { Garcia-Romeu \& } \\
\text { Richards, } 2018\end{array}$ & $\begin{array}{l}\text { Current perspectives on psychedelic } \\
\text { therapy: use of serotonergic } \\
\text { hallucinogens in clinical interventions }\end{array}$ & $\begin{array}{l}\text { Fornecer uma breve visão geral das maneiras pelas } \\
\text { quais o receptor de serotonina } 2 \mathrm{~A} \text { (5-HT2A) } \\
\text { alucinógenos agonistas (ou seja, psicodélicos) } \\
\text { foram usados para fins clínicos ou de pesquisa } \\
\text { como parte de intervenções terapêuticas }\end{array}$ \\
\hline 2 & Hartogsohn, 2018 & $\begin{array}{l}\text { The meaning-enhancing properties of } \\
\text { psychedelics and their mediator } \\
\text { role in psychedelic therapy, } \\
\text { spirituality, and creativity }\end{array}$ & $\begin{array}{c}\text { Defender a importância de outro mediador de ação } \\
\text { psicodélica, que é fundamental para compreender } \\
\text { os efeitos dos psicodélicos em terapia, criatividade } \\
\text { e espiritualidade. }\end{array}$ \\
\hline 3 & Johnson, 2018 & $\begin{array}{l}\text { Psychiatry might need some } \\
\text { psychedelic therapy }\end{array}$ & $\begin{array}{l}\text { Determinar a evolução e estado atual da psiquiatria } \\
\text { e como a terapia psicodélica pode ser útil. }\end{array}$ \\
\hline 4 & $\begin{array}{l}\text { Moreton, Szalla, } \\
\text { Menzies \& Arena } \\
\text { (2019) }\end{array}$ & $\begin{array}{l}\text { Embedding existential psychology } \\
\text { within psychedelic science: } \\
\text { reduced death anxiety as a } \\
\text { mediator of the therapeutic effects } \\
\text { of psychedelics }\end{array}$ & $\begin{array}{l}\text { Fornecer uma revisão complementar dos } \\
\text { mecanismos pelos quais os psicodélicos podem } \\
\text { reduzir a ansiedade da morte }\end{array}$ \\
\hline 5 & $\begin{array}{l}\text { Noorani, Garcia- } \\
\text { Romeu, Swift, } \\
\text { Griffiths \& } \\
\text { Johnson (2018) }\end{array}$ & $\begin{array}{l}\text { Psychedelic therapy for smoking } \\
\text { cessation: qualitative analysis of } \\
\text { participant accounts }\end{array}$ & $\begin{array}{l}\text { Identificar os mecanismos de mudança percebidos } \\
\text { que levam à cessação do tabagismo no estudo } \\
\text { piloto }\end{array}$ \\
\hline 6 & Ross, 2018 & $\begin{array}{l}\text { Therapeutic use of classic psychedelics } \\
\text { to treat cancer-related psychiatric } \\
\text { distress }\end{array}$ & $\begin{array}{l}\text { Identificar a prevalência e impacto adverso } \\
\text { psicológico, existencial e médico de um diagnóstico } \\
\text { de câncer e como a terapia psicodélica pode ser útil }\end{array}$ \\
\hline 7 & Sessa, 2017 & $\begin{array}{c}\text { Why mdma therapy for alcohol use } \\
\text { disorder? And why now? }\end{array}$ & $\begin{array}{l}\text { Propor em um estudo em andamento no Reino } \\
\text { Unido que pacientes em } \\
\text { transtorno de uso de álcool que passaram por uma } \\
\text { desintoxicação médica podem se beneficiar de um } \\
\text { curso de psicoterapia assistida por MDMA }\end{array}$ \\
\hline
\end{tabular}

Fonte: Dados da pesquisa (2021).

No segundo quadro foram expostos o tipo de estudo e seus principais resultados. Quanto ao tipo de estudo desenvolvido pelos artigos analisados, 5 deles se tratavam de revisão bibliográfica, 1 de estudo transversal e 1 de revisão sistemática. 
Quadro 2: Distribuição dos estudos analisados quanto a população e amostra e os principais resultados.

\begin{tabular}{|c|c|c|c|}
\hline $\mathbf{N}^{\circ}$ & $\begin{array}{l}\text { AUTOR/ } \\
\text { ANO }\end{array}$ & $\begin{array}{l}\text { TIPO DE } \\
\text { ESTUDO }\end{array}$ & PRINCIPAIS RESULTADOS \\
\hline 1 & $\begin{array}{l}\text { Garcia-Romeu; } \\
\text { Richards, } 2018\end{array}$ & $\begin{array}{c}\text { Revisão } \\
\text { bibliográfica }\end{array}$ & $\begin{array}{l}\text { Esse estudo contribuiu com percepções seminais sobre como essas } \\
\text { substâncias podem ser empregadas com eficácia e segurança em interações } \\
\text { terapêuticas direcionadas, incluindo a importância de otimizar o conjunto } \\
\text { (estado de espírito) e o ambiente. }\end{array}$ \\
\hline 2 & Hartogsohn, 2018 & $\begin{array}{c}\text { Revisão } \\
\text { bibliográfica }\end{array}$ & $\begin{array}{l}\text { O realce da significação dos psicodélicos é uma hipótese apoiada por } \\
\text { diversos relatos de experiência, bem como pela pesquisa clínica, mas ainda } \\
\text { não recebeu a atenção que merece. Este caminho de investigação pode ser } \\
\text { aberto através do emprego de várias ferramentas psicométricas para auxiliar } \\
\text { na avaliação do grau em que os psicodélicos aumentam o seu significado e } \\
\text { possíveis correlações com terapêuticas espirituais. }\end{array}$ \\
\hline 3 & Johnson, (2018) & $\begin{array}{c}\text { Revisão } \\
\text { bibliográfica }\end{array}$ & $\begin{array}{c}\text { O campo psiquiátrico se encontra relativamente estagnado, não tendo } \\
\text { acompanhado a evolução do restante da medicina. A terapia psicodélica tem } \\
\text { demonstrado resultados promissores e pode representar novas perspectivas na } \\
\text { psiquiatria. }\end{array}$ \\
\hline 4 & $\begin{array}{l}\text { Moreton, Szalla, } \\
\text { Menzies \& Arena } \\
\text { (2019) }\end{array}$ & $\begin{array}{l}\text { Revisão } \\
\text { bibliográfica }\end{array}$ & $\begin{array}{c}\text { O estudo mostra que a psicopatologia pode surgir de tentativas falhas de lidar } \\
\text { com a morte subjacente. Também foi evidenciada a capacidade dos } \\
\text { psicodélicos na ajuda com as questões sobre a mortalidade envolvendo } \\
\text { muitos de seus efeitos terapêuticos até mesmo em indivíduos sem doenças } \\
\text { com risco de vida. }\end{array}$ \\
\hline 5 & $\begin{array}{l}\text { Noorani, Garcia- } \\
\text { Romeu, Swift, } \\
\text { Griffiths \& Johnson } \\
\quad(2018)\end{array}$ & $\begin{array}{l}\text { Estudo de } \\
\text { Coorte } \\
\text { retrospectivo }\end{array}$ & $\begin{array}{l}\text { Os participantes relataram obter percepções vívidas sobre a identidade } \\
\text { própria e as razões para parar de fumar em suas sessões de psilocibina. O } \\
\text { aconselhamento preparatório, e o forte relacionamento com a equipe do } \\
\text { estudo foi percebido como um fator adicional vital para alcançar a } \\
\text { abstinência. Além disso, os participantes relataram uma série de mudanças } \\
\text { positivas além da cessação do tabagismo, incluindo aumento da apreciação } \\
\text { estética, altruísmo e comportamento pró-social. }\end{array}$ \\
\hline 6 & Ross, (2018) & $\begin{array}{c}\text { Revisão } \\
\text { sistemática da } \\
\text { literatura }\end{array}$ & $\begin{array}{c}\text { Esta revisão identificou } 10 \text { ensaios publicados, nos quais psicodélicos } \\
\text { serotoninérgicos foram estudados no tratamento de doenças psiquiátricas } \\
\text { relacionadas ao câncer. Seis ensaios clínicos publicados onde foram incluídos } \\
341 \text { participantes (quase exclusivamente com diagnóstico de câncer e } \\
\text { doenças psicológicas e existenciais relacionadas estresse) tratada com terapia } \\
\text { psicodélica assistida. }\end{array}$ \\
\hline 7 & Sessa, (2017) & $\begin{array}{c}\text { Revisão } \\
\text { bibliográfica }\end{array}$ & $\begin{array}{l}\text { O MDMA tem o potencial de melhorar e intensificar os processos } \\
\text { psicoterapêuticos no tratamento do transtorno do uso de álcool. Também } \\
\text { pode abordar sintomas de outras condições que são frequentemente } \\
\text { comórbidas com transtornos por uso de substâncias, particularmente aqueles } \\
\text { sintomas associados a uma história de trauma psicológico. }\end{array}$ \\
\hline
\end{tabular}

Fonte: Dados da pesquisa (2021).

Conforme apresentado no Quadro 3, todos os estudos $(\mathrm{N}=7)$ destacam benefícios da terapia psicodélica no manejo de pacientes que possuem algum distúrbio psiquiátrico, tais como depressão, transtornos do humor, de ansiedade, estresse póstraumático e na melhora da dependência química de algumas substâncias, como álcool, tabaco, cocaína e opioides. Tais achados são os mais relevantes desse estudo, pontuando os principais benefícios com o tratamento da terapia psicodélica. 
Quadro 3: Principais benefícios da terapia psicodélica.

\begin{tabular}{|c|c|}
\hline AUTOR/ANO & PRINCIPAIS POTENCIAIS BENEFÍCIOS DA TERAPIA PSICODÉLICA \\
\hline $\begin{array}{l}\text { Garcia-Romeu; } \\
\text { Richards, (2018) }\end{array}$ & $\begin{array}{l}\text { Segurança e potencial terapêutico dos psicodélicos (LSD, psilocibina, mescalina e DMT) como } \\
\text { um adjuvante podendo promover transformações substanciais para o tratamento de dependência } \\
\text { química como o alcoolismo, cessação do tabagismo, cocaína, e transtornos como ansiedade, } \\
\text { idealização suicida, dependência em opioides, alterações do humor e até mesmo dor. Além de } \\
\text { aliviar os sintomas clínicos dessas condições, a terapia psicodélica pode também estimular a } \\
\text { criatividade, a espiritualidade e otimizar as funções fisiológicas dos indivíduos. Quando } \\
\text { associado com a terapia de aprimoramento motivacional, os resultados são mais satisfatórios. } \\
\text { Entre as propriedades farmacológicas psicodélicas, destacam-se sua capacidade de evocar } \\
\text { experiências altamente significativas que se prestam a intervenções estruturadas destinadas a } \\
\text { alterar ou modular o pensamento, humor ou padrões de comportamento de um indivíduo por } \\
\text { meio de terapia hábil. }\end{array}$ \\
\hline Hartogsohn, (2018) & $\begin{array}{l}\text { Experiências espiritualmente significativas e experiências de dissolução do ego, cuja ocorrência } \\
\text { é frequentemente correlacionada com o sucesso da terapia. Essas substâncias possuem uma } \\
\text { tendência notável para aumentar a percepção de significado, ou, em outras palavras, para fazer } \\
\text { com que as coisas pareçam ser dramaticamente mais significativas do que poderiam ser. Assim, } \\
\text { a terapia psicodélica possui benefícios na melhora dos sintomas para pacientes com algum } \\
\text { transtorno psiquiátrico ou com dependência química em substâncias como álcool, tabaco e } \\
\text { cocaína. }\end{array}$ \\
\hline Johnson, (2018) & $\begin{array}{l}\text { Potenciais efeitos antidepressivos e ansiolíticos substanciais para pacientes com comorbidades } \\
\text { psicológicas ou orgânicas, tais como câncer. Além disso, a terapia psicodélica contribui } \\
\text { significativamente no tratamento de pacientes com alguma dependência química, álcool, tabaco e } \\
\text { cocaína, tendo influência da mente e no comportamento dos pacientes. }\end{array}$ \\
\hline $\begin{array}{l}\text { Moreton, Szalla, } \\
\text { Menzies \& Arena } \\
\text { (2019) }\end{array}$ & $\begin{array}{l}\text { A terapia psicodélica pode reduzir transtornos de ansiedade associados ou não com ideação } \\
\text { suicida, agindo ainda na dissolução do ego, admiração e conexão dos pacientes com outras } \\
\text { pessoas, além de também promover melhoria em outros transtornos psicológicos, bem como no } \\
\text { tratamento de dependência em algumas substâncias químicas. Além disso, a terapia psicodélica } \\
\text { pode ajudar a esclarecer os mecanismos psicológicos que sustentam os efeitos terapêuticos dos } \\
\text { psicodélicos e o papel desempenhado pela ansiedade da morte na etiologia da doença mental. }\end{array}$ \\
\hline $\begin{array}{l}\text { Noorani, Garcia- } \\
\text { Romeu, Swift, } \\
\text { Griffiths \& Johnson } \\
\quad(2018)\end{array}$ & $\begin{array}{l}\text { Auxílio no tratamento da abstinência alcóolica e de dependência química, cessação do } \\
\text { tabagismo, cocaína, além da redução de estresse psicológico e de ideação suicida em pacientes } \\
\text { psiquiátricos. Mudaças no comportamento, humor e atitudes na personalidade dos indivíduos. }\end{array}$ \\
\hline Ross, (2018) & $\begin{array}{l}\text { O uso de substâncias psicodélicas para o tratamento de pacientes com transtornos psiquiátricos, } \\
\text { tais como depressão e ansiedade, transtorno existencial e de espiritualidade devido a } \\
\text { comorbidades que ameacem a vida, como o câncer, mostrou-se benéfico na diminuição dos } \\
\text { sintomas depressivos e de ansiedade, associados ou não com o câncer. }\end{array}$ \\
\hline Sessa, (2017) & $\begin{array}{l}\text { O uso da terapia psicodélica pode amenizar sintomas do transtorno do estresse pós-traumático } \\
\text { (TEPT) e também o de dependência de substâncias como álcool, tabaco, cocaína e opioides ou } \\
\text { outras drogas que possam ocasionar dependência química. A associação com a terapia de } \\
\text { aprimoramento motivacional pode potencializar os efeitos. }\end{array}$ \\
\hline
\end{tabular}

Fonte: Dados da pesquisa (2021).

De acordo com os dados do Quadro 4, todos os estudos selecionados ( $\mathrm{N}=7$ ) identificaram forte correlação entre o uso da terapia psicodélica assistida e a melhora dos sintomas em pacientes com algum transtorno psiquiátrico (depressão bipolar maior, humor, ansiedade e estresse pós-traumático) e dependência química em algumas substâncias, tais como álcool, tabaco, cocaína, opioides e outras substâncias. 
Quadro 4: Categorização dos principais benefícios da terapia psicodélica em relação aos âmbitos psiquiátrico e dependência química.

\begin{tabular}{|l|c|}
\hline $\begin{array}{c}\text { PRINCIPAIS BENEFÍCIOS DA TERAPIA } \\
\text { PSICODÉLICA EM RELAÇÃO AO ÂMBITO: }\end{array}$ & $\begin{array}{c}\text { FREQUÊNCIA DOS ACHADOS NOS } \\
\text { ESTUDOS (\%) }\end{array}$ \\
\hline $\begin{array}{l}\text { Psiquiátrico (transtornos depressivos, do humor, } \\
\text { ansiedade, estresse pós-traumático) }\end{array}$ & $\mathrm{N}=7(100 \%)$ \\
\hline $\begin{array}{l}\text { Dependência química (álcool, tabaco, cocaína, } \\
\text { opioides e outras substâncias químicas que possam } \\
\text { ocasionar dependência) }\end{array}$ & $\mathrm{N}=7(100 \%)$ \\
\hline
\end{tabular}

Fonte: Dados da pesquisa (2021).

\section{Discussão}

O termo "psicodélico" e "psicodelia" foram cunhados em 1957 pelo psiquiatra britânico Humphry Osmond, numa troca de cartas com o escritor Aldous Huxley, em 1956. Psicodelia traduz a ideia de manifestação da mente, ou revelação do espírito, e psicodélico é o que torna visível a alma, o self ou o psiquismo (Rodrigues, 2019).

Embora o termo fosse novo, os psicodélicos já eram usados há milênios em rituais religiosos e contextos sagrados na América central (Hofemann, 2013). O sentido de psicodelia, no sentido que interessa à clínica, refere-se a experiências de transformação por redução de controle egóico e expansão da consciência (Rodrigues, 2019).

Porém no início do uso clínico dos psicodélicos, os psiquiatras não se concentravam na capacidade terapêutica da substância, mas tentavam, principalmente, induzir psicoses (Grof, 1997).

Quanto ao título e objetivo dos artigos, Garcia-Romeu e Richards (2018) procuraram falar sobre as perspectivas atuais sobre terapia psicodélica e o uso de alucinógenos em intervenções; Hartogsohn (2018) abordou sobre as propriedades dos psicodélicos e seu papel na terapia psicodélica, espiritualidade e criatividade; Johnson (2018) falou sobre como a psiquiatria pode precisar da terapia psicodélica; Moreton et al. (2019) estudou a incorporação da psicologia existencial na ciência psicodélica e a redução da ansiedade da morte através dos efeitos psicodélicos; Noorani et al. (2018) buscou estudar a terapia psicodélica para o processo de cessação o tabagismo; Ross (2018) avaliou o uso terapêutico de psicodélicos para o tratamento em pacientes com câncer e, por fim, Sessa (2017) investigou a terapia com MDMA para os transtornos de abuso de álcool.

Foi a partir da difusão da Dietilamida do Ácido Lisérgico (LSD) que o potencial transformacional dos Psicodélicos se tornou obvio e o seu uso terapêutico teve então início (Grof, 1997). Assim, nas décadas de 1950 e 1960 vários estudos foram feitos acerca do potencial terapêutico dessas substâncias, como para fins de tratamento de estresse pós traumático, depressão e para incursão em sessões de psicanálise (Grispoon \& Bakalar, 1979). Porém com a Lei de substâncias controladas de 197, esse campo de pesquisas praticamente cessou (Daniel \& Haberman, 2017).

Garcia-Romeu e Richards (2018) constataram que a terapia psicodélica por mais antiga que seja, viu um renascimento encorajador com ensaios clínicos promissores no início do século 21. Para eles, isso acontece apoiado pela quebra de tabus e estigmas da sociedade nos últimos 20 anos e, também, pela relativa letargia e baixa resolução na psiquiatria desde os anos 70 se comparado ao restante da medicina. Que vê nelas uma esperança para alguns dos problemas mais difíceis da psiquiatria.

Johnson (2018) acredita que a psiquiatria precisa de ajuda. Ele alerta para as taxas epidêmicas de suicídio e as milhões de mortes causadas anualmente pelo vício em tabaco. Ainda lembra que o último grande avanço para tratar depressão foi há mais de 30 anos com a aprovação do primeiro ISRS (inibidor seletivo da receptação de serotonina) e que, mesmo estes, eram evoluções simples mais seguras e seletivas de antidepressivos já desenvolvidos na década de 1950. 
Ainda segundo Johnson (2018), é importante ressaltar que esses medicamentos, mesmo com efeitos relativamente modestos, são cruciais para aqueles cuja depressão os põem em risco de suicídio, possibilitando uma necessidade clara e um espaço substancial para melhorias. Para ele, os avanços lentos e modestos na psiquiatria estão relacionados com a falta de mecanismos analíticos de várias doenças e, nesse sentido, os psicodélicos poderiam ser de grande importância para fazer “avanços fundamentais em uma mecânica compreensão (biológica e psicológica) de distúrbios psiquiátricos”.

Noorani et al. (2018) fizeram uma análise qualitativa retrospectiva de entrevistas feitas com participantes de estudos pilotos para cessação do tabagismo facilitada por psilocibina. Segundo eles, embora esses estudos não possam demonstrar a eficácia, eles convergem com uma gama de outros resultados que indicam um significativo poder terapêutico dos alucinógenos para tratar o tabagismo. Da análise eles concluíram que, além da cessação do tabagismo, a terapia proporcionou outros efeitos positivos persistentes como autoestima e altruísmo.

Ross (2018) fez uma revisão sistemática da literatura sobre o uso clínico de alucinógenos para tratar o sofrimento psicológico relacionado câncer. Eles lembram que houve uma fase inicial promissora de pesquisas clínicas com alucinógenos entre 1960 e 1970, e que elas foram interrompidas antes que qualquer conclusão definitiva fosse alcançada. Mas que na última década os ensaios clínicos foram retomados para o tratamento de várias doenças psiquiátricas, das quais ele cita: o sofrimento relacionado ao câncer; depressão; toc; além de transtornos de dependência (álcool, nicotina e cocaína), sendo o uso de alucinógenos clássicos para o sofrimento relacionado ao câncer do qual se tem dados mais robustos.

Para Ross (2018), os ensaios clínicos revisados têm resultados significativamente positivos e demostram ter relação com aceitação, reflexão de morte e enriquecimento espiritual, o que seriam pontos chaves do sofrimento pela ansiedade com a própria morte.

Moreton et al. (2019) abordam a psicologia existencial na ciência psicodélica. Eles argumentam que ansiedade da morte tem um papel central na psicopatologia e que os psicodélicos em uma correlação de fatores como: confronto de medos inconscientes de morte; redução do foco no eu; mudanças de percepção de consciência humana; aumento da fé e ampliações de significado da vida promovem redução da ansiedade da morte. Para eles, os psicoterapeutas devem voltar seus olhos para o consciente/inconsciente como solução para a estagnação notada na psicoterapia e a ciência psicodélica estaria para isso como uma 'superestrada'.

Hartogsohn (2018) investigou as aparentes propriedades de aumento de significado dos psicodélicos. O autor encontrou em estudos feitos com questionários um aumento de significado para a vida e questões existenciais em pacientes que fizeram uso de psilocibina. Para ele, isso acontece em função de uma demanda humana espontânea em atribuir significado e que os psicodélicos, por meio de um poderoso acesso ao que antes era subconsciente, permite a criação de novos conceitos e definições e pode auxiliar na terapia, espiritualidade e criatividade.

Sessa (2017) propõem que um estudo em andamento com pacientes que passaram por desintoxicação médica para o transtorno de abuso de álcool pode ter benefícios fazendo uso da terapia com MDMA. Para justificar esse ponto de vista, o autor argumenta que o MDMA é mais seguro que o restante dos psicodélicos e que seu uso já demonstrou em outras ocasiões efeitos animadores para o transtorno por abuso de álcool e traumas não solucionados. Para finalizar, argumenta também que a sociedade e a psiquiatria estão sobrecarregadas por resultados no tratamento do transtorno de abuso de álcool que "são um pouco melhores agora do que eram a 100 anos atrás”.

Dentre as limitações apresentadas para a realização desse estudo, destacam-se a baixa quantidade de estudos realizados sobre a temática, sendo necessários mais ensaios clínicos randomizados e estudos observacionais para auxiliar no melhor entendimento sobre a terapia psicodélica e seus benefícios para a saúde mental dos pacientes. Ainda, há poucos estudos que expliquem os mecanismos de ação farmacocinéticos dos psicodélicos nas vias que regulam e modulam a neurotransmissão relacionadas com transtornos do humor. 


\section{Considerações Finais}

Vários dos autores observam o renascimento da terapia psicodélica e encontram na própria psicoterapia razões para tal. Parece haver um descontentamento geral com a resolubilidade atual das psicopatologias e a evolução da psicoterapia na medicina.

A terapia psicodélica é então vista como um novo e promissor horizonte nesse campo que está se aproveitando da quebra de paradigmas na sociedade para avançar nas pesquisas relativas ao tratamento de sérios problemas de saúde como depressão e ansiedade da morte, além de transtornos de dependência como alcoolismo e tabagismo, em que os resultados decorrentes têm sido animadores e de onde os pesquisadores esperam e precisam por avanços significativos.

Esse trabalho procurou abordar brevemente a história da terapia psicodélica e, principalmente, contextualizar as investigações mais recentes que marcam o seu renascimento. Ou seja, essencialmente, apresentar as suas aplicabilidades e potencialidades, bem como a demanda a qual se insere. Não é objetivo desse trabalho determinar a eficácia dos tratamentos ou seus mecanismos de ação. Assim, qualquer conclusão nesse sentido a respeito do que foi aqui tratado não é absolutamente precisa.

Sugere-se para pesquisas futuras o esclarecimento entre a relação entre os alucinógenos com os efeitos terapêuticos biológicos para os indivíduos, os benefícios psicológicos com a terapia psicodélica, bem como destacar os possíveis efeitos adversos dessa modalidade terapêutica. Além disso, o esclarecimento do mecanismo de ação dos psicodélicos na neurotransmissão relacionada com distúrbios psiquiátricos é também necessária para o melhor entendimento de seus benefícios.

\section{Referências}

Brasil (2020). Alucinógenos que podem curar. Scientific American Brasil.

Daniel, J., \& Haberman, M. (2018). Clinical potential of psilocybin as a treatment for mental health conditions. The mental health clinician, 7(1), 24-28. https://doi.org/10.9740/mhc.2017.01.024

Doblin, R. E. (2020). Regulation of the Medical Use of Psychedelics and Marijuana. Harvard. https://www.psychologytoday.com/file s/attachments/90761/doblinharvard2000regulation-the-medical-use-psychedelics-and-marijuana.pdf

Garcia-Romeu, A., \& Richards, W. A. (2018). Current perspectives on psychedelic therapy: use of serotonergic hallucinogens in clinical interventions. International review of psychiatry (Abingdon, England), 30(4), 291-316. https://doi.org/10.1080/09540261.2018.1486289

Gardner, J., Carter, A., O'brien, K., \& Seear, K. (2019). Psychedelic-assisted therapies: the past, and the need to move forward responsibly. International Journal Of Drug Policy, 70X), 94-98, 2019. http://dx.doi.org/10.1016/j.drugpo.2019.05.019.

Grinspoon, L., \& Bakalar, J. (1997). Psychedelic drugs reconsidered. New York Lindesmith Center.

Grof, S. (1997). Os psicodélicos na autoexploração e na psicoterapia. Associação Psicodélica do Brasil.

Hartogsohn I. (2018). The Meaning-Enhancing Properties of Psychedelics and Their Mediator Role in Psychedelic Therapy, Spirituality, and Creativity. Frontiers in neuroscience, 12, 129. https://doi.org/10.3389/fnins.2018.00129

Hofemann, A. (2017) LSD: My problem child 4th edition. Multidisciplinary Association for Psychedelic Studies.

James, W. (2017). As variedades da experiência religiosa. São Paulo: Cultrix.

Johnson M. W. (2018). Psychiatry might need some psychedelic therapy. International review of psychiatry (Abingdon, England), 30(4), 285-290. https://doi.org/10.1080/09540261.2018.1509544

Leary, T. (1983). Flashbacks: An Autobiography. Los Angeles: J.P. Tarcher.

Luoma, J. B., Sabucedo, P., Eriksson, J., Gates, N., \& Pilecki, B. C. (2019). Toward a contextual psychedelic-assisted therapy: perspectives from acceptance and commitment therapy and contextual behavioral science. Journal Of Contextual Behavioral Science, 14(X), 136-145. http://dx.doi.org/10.1016/j.jcbs.2019.10.003.

Moreton, S. G., Szalla, L., Menzies, R. E., \& Arena, A. F. (2020). Embedding existential psychology within psychedelic science: reduced death anxiety as a mediator of the therapeutic effects of psychedelics. Psychopharmacology, 237(1), 21-32. https://doi.org/10.1007/s00213-019-05391-0 
Research, Society and Development, v. 10, n. 9, e48510918122, 2021

(CC BY 4.0) | ISSN 2525-3409 | DOI: http://dx.doi.org/10.33448/rsd-v10i9.18122

Noorani, T., Garcia-Romeu, A., Swift, T. C., Griffiths, R. R., \& Johnson, M. W. (2018). Psychedelic therapy for smoking cessation: Qualitative analysis of participant accounts. Journal of psychopharmacology (Oxford, England), 32(7), 756-769. https://doi.org/10.1177/0269881118780612

Rodrigues, S. (2016). Modulações de sentidos na experiência psicodélica: saúde mental e gestão autônoma de psicotrópicos prescritos e proscritos. Curitiba: $C R V$

Ross S. (2018). Therapeutic use of classic psychedelics to treat cancer-related psychiatric distress. International review of psychiatry (Abingdon, England), 30(4), 317-330. https://doi.org/10.1080/09540261.2018.1482261

Sessa B. (2018). Why MDMA therapy for alcohol use disorder? And why now? Neuropharmacology, 142, 83-88. https://doi.org/10.1016/j.neuropharm.2017.11.004

Souza, M. T. de, Silva, M. D. da, \& Carvalho, R. de. (2010) Integrative review: what is it? how to do it? Einstein (São Paulo), 8(1), 102-106. http://dx.doi.org/10.1590/s1679-45082010rw1134.

Strassman, R. (2000). DMT - the spirit molecule: a doctor's revolutionary research into the biology of near-death and mystical experiences. Rochester: Park Street Press.

Strassman R. J. (1996). Human psychopharmacology of N,N -dimethyltryptamine. Behavioural brain research, 73(1-2), 121-124. https://doi.org/10.1016/0166-4328(96)00081-2

Watts, R., \& Luoma, J. B. (2020). The use of the psychological flexibility model to support psychedelic assisted therapy. Journal Of Contextual Behavioral Science, 15(X), 92-102.http://dx.doi.org/10.1016/j.jcbs.2019.12.004. 